\title{
Differential outcomes and many-to-one matching: Effects of correlation with correct choice
}

\author{
PETER J. URCUIOLI \\ Purdue University, West Lafayette, Indiana
}

\begin{abstract}
Pigeons trained on many-to-one matching-to-sample with different probabilities of reinforcement for correct choice acquired the task more rapidly and showed better working memory performance when those different probabilities were correlated with the correct comparisons than when they were uncorrelated with them. Furthermore, this advantage was maintained when all correct choices were subsequently reinforced at the same probability, even though the change to nondifferential outcomes produced a drop in accuracy in the (formerly) correlated group. When birds were later shifted from correlated to uncorrelated outcomes or vice versa, the original between-group differences were reversed or eliminated. These data suggest that differential outcomes will potentiate matching performance if they generate an expectancy cue that is also predictive of correct choice. In addition, different outcomes may enhance common coding of the samples in many-to-one matching to the extent that they too are correlated with the correct comparison alternatives.
\end{abstract}

One of the most consistent and powerful effects on the learning and retention of conditional discriminations is the enhancement of performance by differential outcomes. For example, pigeons acquire two-alternative matchingto-sample (MTS) much more rapidly when the correct choice following each sample stimulus produces a unique outcome than when it produces either the same outcome on all trials or each outcome equally often (Brodigan \& Peterson, 1976; Edwards, Jagielo, Zentall, \& Hogan, 1982; Linwick, Overmier, Peterson, \& Mertens, 1988; Peterson, 1984; Peterson, Linwick, \& Overmier, 1987; Peterson, Wheeler, \& Trapold, 1980; Santi \& Roberts, 1985a; Urcuioli, 1990; Williams, Butler, \& Overmier, 1990). The same effect also occurs in related tasks such as successive matching (DeLong \& Wasserman, 1981; Honig, Matheson, \& Dodd, 1984; see also Urcuioli \& Zentall, 1990). My purpose in the present study was to investigate the processes by which this differentialoutcomes effect (DOE) might be produced.

The DOE has been frequently interpreted to mean that subjects can anticipate early in a trial the particular outcome scheduled to occur later in the trial (Honig \& Thompson, 1982; Peterson, 1984; Trapolu, 1970). By this argument, the DOE could occur either because there are two potential cues (i.e., samples plus outcome expectancies) for performance as opposed to only one, or because outcome expectancies provide a much more salient (and hence dominant) source of control than the nominal dis-

\footnotetext{
This research was supported by NSF Grant $86-06926$. The author thanks Beth Kraemer, Nicholas Ketterer, and Judy Tedlie for running subjects. Correspondence concerning this paper should be addressed to Peter J. Urcuioli, Department of Psychological Sciences, Purdue University, West Lafayette, IN 47907.
}

criminative stimuli themselves (Urcuioli, 1990; cf. Urcuioli, 1985).

In either case, this analysis assumes that another discriminative cue is in fact generated by the association between samples and outcomes. This assumption has been independently verified in other experiments, the most compelling of which have been transfer-of-control studies. In these experiments, the different outcomes used in MTS are also associated with other, off-baseline stimuli. Later, those stimuli are substituted for the samples as conditional cues for matching. This substitution yields positive transfer of performance when the off-baseline stimuli have the same outcome associates as the samples they replace (Peterson, 1984; Urcuioli, 1990; see also Edwards et al., 1982; and Honig et al., 1984). By contrast, negative transfer is found when those stimuli have different outcome associates (Edwards et al., 1982). These results, then, support outcome-expectancy mediation of the DOE.

Nevertheless, other mechanisms could also underlie or contribute to the effect. For example, the samples might be more discriminable when they are associated with different, rather than identical, outcomes (cf. Mackintosh, 1983). According to this account, the DOE is essentially another instance of "acquired distinctiveness of cues" (Lawrence, 1949; cf. Peterson \& Trapold, 1982). In other words, pairing different outcomes with the sample stimuli increases the associability of those samples with the correct comparison alternatives. Consequently, the rate with which the samples acquire conditional stimulus control and/or the degree to which they exert that control in steady-state behavior is potentiated (cf. Wright \& Sands, 1981).

The relative contributions of enhanced sample discriminability versus outcome-expectancy mediation can be evaluated in part by the experimental design shown 
in Table 1. Here, separate groups are trained on MTS in which different outcomes $\left(\mathrm{O}_{\mathrm{x}}\right.$ and $\left.\mathrm{O}_{\mathrm{y}}\right)$ are associated with the sample stimuli $\left(S_{1}, S_{2}, S_{3}\right.$, and $\left.S_{4}\right)$. Given this arrangement, sample discriminability should presumably be equated across groups. For one group, however, the different outcomes are perfectly correlated with the different correct choices $\left(C_{1}\right.$ vs. $\left.C_{2}\right)$. Consequently, any outcome expectancies $\left(E_{x}\right.$ and $\left.E_{y}\right)$ arising from the sample-outcome associations in this group can provide an additional cue for choice. By contrast, outcomes are uncorrelated with correct choice in the remaining group, so for them, matching performances cannot simply be cued by the expectancies alone. If the two groups acquire their tasks with equal rapidity, this would support the argument that enhanced sample discriminability underlies the DOE (assuming, of course, that both groups learn faster than a group trained with nondifferential outcomes). On the other hand, if the correlated group acquires MTS more quickly than the uncorrelated group, this would indicate that the DOE is attributable to the discriminative properties of a valid expectancy cue.

Surprisingly, these two accounts have been contrasted in only one study in the differential outcomes literature. Using a design like that shown in Table 1, Honig et al. (1984) compared how rapidly pigeons acquired a foursample successive matching task (cf. Nelson \& Wasserman, 1978) as a function of whether food versus water outcomes were correlated or uncorrelated with go/no-go responding to the test (comparison) stimuli. They found faster matching acquisition with correlated than with uncorrelated training, a result consistent with outcomeexpectancy mediation. One of the purposes of the present study was to test the generality of this finding by determining whether or not the differences reported by Honig et al. (1984) would occur in two-alternative MTS, a task commonly used in differential-outcomes research.

A second purpose of the present study was to provide a separate assessment of conditional stimulus control by the samples after the training task had been acquired to high levels of accuracy. Again, different predictions can be derived from the sample discriminability versus outcome-expectancy accounts. If differential outcomes merely enhance sample-stimulus control, then the samples should support approximately the same level of ac-

Table 1

A Design for Comparing Enhanced Sample Discriminability Versus Outcome-Expectancy Mediation as Factors Producing the Differential Outcomes Effect

\begin{tabular}{cl}
\hline Group & Matching Task \\
\hline Correlated & $S_{1} \cdot\left(E_{x}\right) \rightarrow C_{1}\left(O_{x}\right)$ \\
& $S_{2} \cdot\left(E_{y}\right) \rightarrow C_{2}\left(O_{y}\right)$ \\
& $S_{3} \cdot\left(E_{x}\right) \rightarrow C_{1}\left(O_{x}\right)$ \\
& $S_{4} \cdot\left(E_{y}\right) \rightarrow C_{2}\left(O_{y}\right)$ \\
& $S_{1} \cdot\left(E_{x}\right) \rightarrow C_{1}\left(O_{x}\right)$ \\
Uncorrelated & $S_{2} \cdot\left(E_{y}\right) \rightarrow C_{2}\left(O_{y}\right)$ \\
& $S_{3} \cdot\left(E_{y}\right) \rightarrow C_{1}\left(O_{y}\right)$ \\
& $S_{4} \cdot\left(E_{x}\right) \rightarrow C_{2}\left(O_{x}\right)$ \\
\hline
\end{tabular}

curacy following correlated and uncorrelated training. By contrast, if those outcomes generate expectancies that provide a salient and valid cue for choice, that cue might reduce the control exerted by the samples relative to when the latter provide the only valid cue for choice (cf. Wagner, Logan, Haberlandt, \& Price, 1968). In other words, conditional stimulus control by the samples could be overshadowed by outcome expectancies that are equally predictive of correct choice (Honig et al., 1984; Urcuioli, 1990).

\section{EXPERIMENT 1}

In the first experiment, separate groups of pigeons were trained on many-to-one MTS (Urcuioli, Zentall, JacksonSmith, \& Steirn, 1989) with four samples, two comparisons, and different probabilities of reinforcement as differential outcomes (Santi \& Roberts, 1985b). For one group, those probabilities were associated with the samples in such a way that any outcome expectancies that might develop could serve as discriminative cues for choice. For a second group, the assignment of reinforcement probabilities to samples ensured that outcome expectancies alone could not cue which choice to make. All birds were initially run in their respective conditions to high and stable levels of accuracy with a 0-sec delay separating samples from comparisons. Afterwards, retention tests were conducted in which four different delays were presented during each session. These tests were run to obtain more extensive data than those reported by Honig et al. (1984) on the effects of correlated versus uncorrelated outcomes on working memory performance.

If the DOE is primarily mediated by the presence of an outcome expectancy cue (as suggested by Honig et al., 1984), then the correlated group should acquire MTS faster, and show better retention, than the uncorrelated group. On the other hand, if differential outcomes facilitate performance primarily by making the samples more distinctive, then the rate of matching acquisition and retention performances should be similar in the two groups.

\section{Method}

Subjects. Eight experimentally naive White Carneaux pigeons served as subjects. The birds were retired breeders obtained from the Palmetto Pigeon Plant (Sumter, SC) and were housed individually in wire-mesh cages in a colony room on a $14: 10$ - $\mathrm{h}$ light:dark cycle. Grit and water were available at all times in the home cage. Prior to the experiment, the birds were gradually reduced by restricted feeding to $80 \%$ of their free-feeding body weights. They were maintained within $\pm 15 \mathrm{~g}$ of that level throughout the experiment by adjusting reinforcement times in the apparatus on a daily basis. Supplemental home-cage feedings were rarely given, although the birds were fed their daily food ration the 1 day/week they were not run. Prior to the start of the experiment, the birds were grouped into pairs on the basis of similar body weights. Members of each pair were then randomly assigned to the two groups described below.

Apparatus. The experiment was run in a standard BRS/LVE three-key pigeon chamber (Model PIP-016 intelligence panel inside Model SEC-002 enclosure). Three circular pecking keys, $2.5 \mathrm{~cm}$ in diameter, were mounted in a row $10 \mathrm{~cm}$ from the top of the intelligence panel and spaced $8.3 \mathrm{~cm}$ center to center. Be- 
hind each was an in-line stimulus projector (Model IC-901-IDD) that could project three white vertical or horizontal lines on a black background, as well as blue, yellow, red, green, and white hues (Pattern No. 692). The $5 \times 5.8 \mathrm{~cm}$ opening for the rear-mounted food magazine was positioned approximately $13 \mathrm{~cm}$ below the center key. The pigeon's compartment inside the enclosure was $37 \times 30$ $\times 34 \mathrm{~cm}$ (height $\times$ width $\times$ depth) and was illuminated by a partially covered GE No. 1829 bulb that directed light toward the ceiling. Ventilation and sound masking was provided by a blower fan attached to the outside of the chamber. Experimental events were controlled and monitored by an IBM XT located in an adjacent room.

Procedure. All birds were first trained to eat reliably from the food hopper, after which they were trained by the method of successive approximations to peck the center key whenever it was lit by a white (W) light. Then, in separate sessions, they received 60 reinforced trials for pecking vertical $(\mathrm{V})$ and horizontal $(\mathrm{H})$ lines, as well as blue (B) and yellow (Y) hues. Finally, all birds received 80 trials of reinforced pecking to all three keys, with red $(R)$ and green $(G)$ each appearing 10 times on the left and right keys, and $\mathrm{V}, \mathrm{H}, \mathrm{B}$, and $\mathrm{Y}$ each appearing 10 times on the center key. Reinforcement in all of these sessions consisted of 3-sec access to mixed grain. Trials were separated by a 10 -sec intertrial interval (ITI), and the houselight was on throughout each session.

Next, the birds received 15 sessions of differential-outcomes "pretraining" with the stimuli that would later serve as samples in MTS. Each of the 60 trials in these sessions began with the onset of a W "ready" stimulus on the center key. The first peck to $\mathrm{W}$ immediately turned it off and produced the trial stimulus ( $\mathrm{V}$, $\mathrm{H}, \mathrm{B}$, or Y) $500 \mathrm{msec}$ later on the same key. The first peck to the line or hue initiated a 3-sec observation period, after which the first keypeck turned the stimulus off and produced food reinforcement if scheduled (a modified fixed-interval or FI 3-sec schedule). For half of the birds, food occurred on all trials with V $(p=1.0)$ but on only $20 \%$ of the trials with $\mathrm{H}(p=.2)$. For the remaining birds, these probabilities were reversed. The same counterbalancing arrangement was used for the $B$ and $Y$ hues. In addition, for the birds assigned to Group Correlated (see below), the reinforcement probabilities associated with $\mathrm{V}$ and $\mathrm{H}$ were the same as those associated with $B$ and $Y$, respectively. For the birds assigned to Group Uncorrelated (see below), they were reversed.

Only the $\mathrm{V}$ and $\mathrm{H}$ lines appeared during the first five pretraining sessions, and only $B$ and $Y$ appeared during the next five. All four stimuli were then presented in each of the last five pretraining sessions. When just the lines or the hues were presented, both stimuli appeared 30 times in pseudorandom order, with the restriction that neither could appear more than three times in a row. When all four stimuli were presented, each appeared 15 times. Successive trials were again separated by a $10-\mathrm{sec}$ ITI, but with the first $9 \mathrm{sec}$ spent in darkness. The houselight was turned on for the last $1 \mathrm{sec}$ of the ITI, and it remained on until the end of the trial, which was defined as the end of the reinforcement cycle or the equivalent period of nonreinforcement. The duration of food reinforcement was constant for individual birds within a session, but it was varied from 2 to $5 \mathrm{sec}$ across sessions to maintain $80 \%$ body weights.

Many-to-one MTS acquisition began on the session following completion of the pretraining phase. Each acquisition session consisted of 96 matching trials, 24 with each center-key (sample) stimulus. Each trial was separated from the preceding one by a 10-sec ITI, the first $9 \mathrm{sec}$ of which were spent in darkness. At that point, the houselight was turned on, and, 1 sec later, the W "ready" stimulus appeared on the center key. Pecking this stimulus immediately turned it off and produced the sample $500 \mathrm{msec}$ later. The first sample keypeck initiated a 3-sec observation period, after which one additional peck turned the sample off and immediately produced $R$ and $G$ comparison hues on the side keys. For all birds, pecking the $\mathrm{R}$ comparison was the correct choice on $\mathrm{V}$ - and B-sample trials,
Table 2

Treatment Conditions for Experiment 1 (Training) and Experiment 2 (Testing)

\begin{tabular}{clll}
\hline Group & & Training & Testing \\
\hline Correlated & $\mathrm{V} \rightarrow \mathrm{R}+$ & $p=1.0$ & $p=.6$ \\
& $\mathrm{H} \rightarrow \mathrm{G}+$ & $p=.2$ & $p=.6$ \\
& $\mathrm{~B} \rightarrow \mathrm{R}+$ & $p=1.0$ & $p=.6$ \\
Uncorrelated & $\mathrm{Y} \rightarrow \mathrm{G}+$ & $p=.2$ & $p=.6$ \\
& $\mathrm{~V} \rightarrow \mathrm{R}+$ & $p=1.0$ & $p=.6$ \\
& $\mathrm{H} \rightarrow \mathrm{G}+$ & $p=.2$ & $p=.6$ \\
& $\mathrm{~B} \rightarrow \mathrm{R}+$ & $p=.2$ & $p=.6$ \\
& $\mathrm{Y} \rightarrow \mathrm{G}+$ & $p=1.0$ & $p=.6$ \\
\hline
\end{tabular}

Note $-\mathrm{V}=$ three white vertical lines on a black background, $\mathrm{H}=$ three white horizontal lines on a black background, $\mathrm{B}=$ blue homogeneous field, $Y=$ yellow homogeneous field, $R=$ red homogeneous field, $G=$ green homogeneous field. Samples appear to the left of the arrows, and correct comparisons appear to the right. Reinforcement probabilities for correct choice during training and testing are shown after the comparisons. Sample-response requirements, incorrect comparisons, and counterbalancing of reinforcement probabilities are omitted for clarity.

and pecking $\mathrm{G}$ was correct on $\mathrm{H}$ - and $\mathrm{Y}$-sample trials. Pecking the alternative comparison on these trials was "incorrect." Each comparison hue appeared equally often on the left and right side keys after each sample.

The two groups differed in the outcomes associated with correct choices on the four trial types, as is shown in Table 2. For Group Correlated, half of the birds received food following every correct comparison choice on trials with the $\mathrm{V}$ and $\mathrm{B}$ samples $(p=1.0)$ but after only $20 \%$ of the correct choices on trials with the $\mathrm{H}$ and Y samples $(p=.2)$. Thus, reinforcement occurred after every correct choice of the $\mathrm{R}$ comparison, but after only $20 \%$ of correct Gcomparison choices for these birds. The opposite contingencies were in effect for the remaining correlated birds. For Group Uncorrelated, half of the birds were reinforced for every correct choice on trials beginning with $\mathrm{V}$ and $\mathrm{Y}$, but for only $20 \%$ of correct choices following $\mathbf{H}$ and $\mathbf{B}$. The remaining birds had the opposite contingencies. For all of the uncorrelated birds, food followed correct $R$ and G-comparison choices $60 \%$ of the time on the average. When birds in either group made a correct but nonreinforced choice response, the houselight stayed on after the comparisons went off for a period equal to the reinforcement duration for that session. Incorrect choices, on the other hand, immediately turned the houselight off for an equivalent period.

Each pair of correlated-uncorrelated birds was run in acquisition until both had chosen the correct comparison on $90 \%$ or more of all trials in five of six consecutive sessions. At that point, both began retention testing with mixed delays.

The 20 sessions of mixed-delay MTS were identical to those described above, except that one of four different retention intervals $(0,1,2$, and $4 \mathrm{sec})$ separated sample offset and comparison onset. Each interval occurred equally often on the eight different trial types defined by the sample and the left-right configuration of the comparisons. All other procedural details for these sessions were identical to those for acquisition.

For all statistical analyses, observed variance ratios from analysis of variance were compared to the tabled values provided by Rodger (1975). The latter ensures that the expected proportion of nulls rejected in error will not exceed $E \alpha$, which was set at .05 .

\section{Results}

Acquisition of many-to-one MTS for each pair of correlated-uncorrelated birds is plotted over blocks of two sessions in Figure 1. Every correlated bird matched at above-chance levels of accuracy and reached criterion 


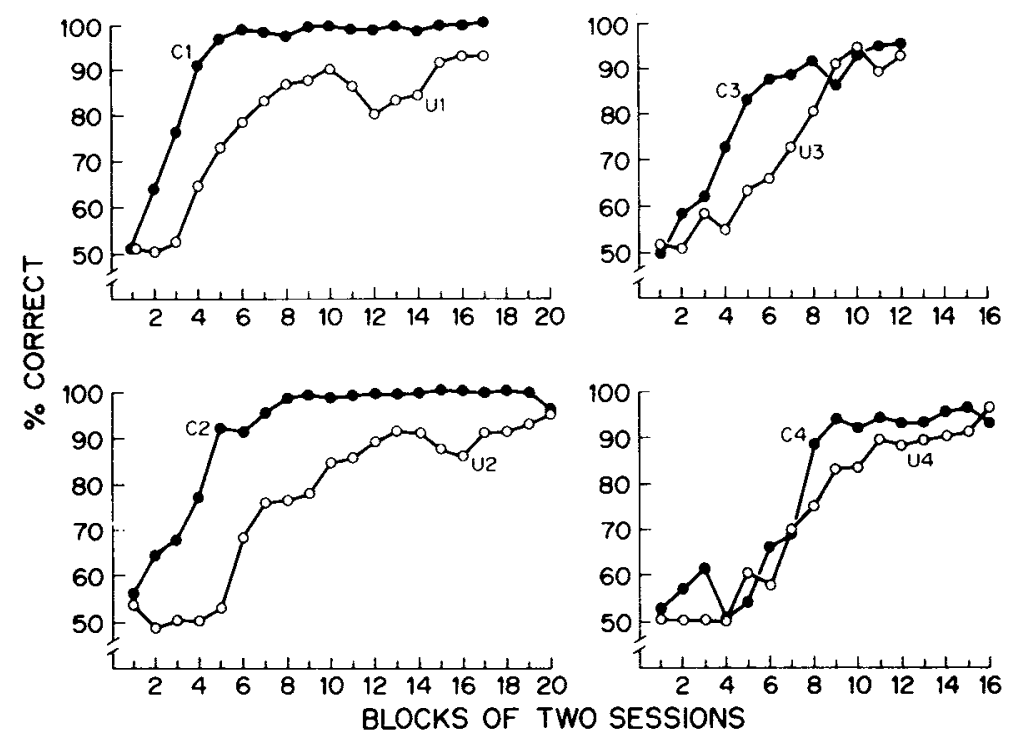

Figure 1. Percentage of correct choice responses over blocks of two acquisition sessions for each pair of correlated and uncorrelated birds in Experiment 1.

levels of performance sooner than its uncorrelated partner. The average number of sessions to reach $90 \%$ accuracy was 12.25 for Group Correlated versus 27.0 for Group Uncorrelated $[F(1,6)=14.94]$.

Accuracy of performance at the end of acquisition also differed between groups. Over the last five sessions, Group Correlated and Group Uncorrelated correctly matched on $97.2 \%$ and $92.1 \%$ of all trials, respectively $[F(1,6)=18.30]$. This difference, however, is probably attributable to overtraining in Group Correlated, since the effect was not evident at the beginning of each bird's criterion "run." When the average percentage correct is computed on the basis of the first five criterion sessions, performances in the two groups were comparable: $92.5 \%$ and $92.1 \%$ for Groups Correlated and Uncorrelated, respectively $[F(1,6)=0.24]$.
The retention gradients obtained from each group following matching acquisition are plotted as the filled symbols in Figure 2. These gradients represent each group's performance averaged over the last 10 mixed-delay sessions with differential (D) outcomes. They show that matching accuracy was higher overall and was less disrupted by increasing delays in Group Correlated than in Group Uncorrelated. An analysis of variance (ANOVA) showed a significant group effect $[F(1,6)=42.79]$ and a significant group $\times$ delay interaction $[F(3,18)=6.90]$. The effect of delay averaged across groups was also significant $[F(3,18)=47.11]$.

During both the acquisition and the mixed-delay phases, the birds in both groups responded differentially to the sample stimuli. The most obvious differential behavior, shown in Table 3, was that the birds took longer to peck

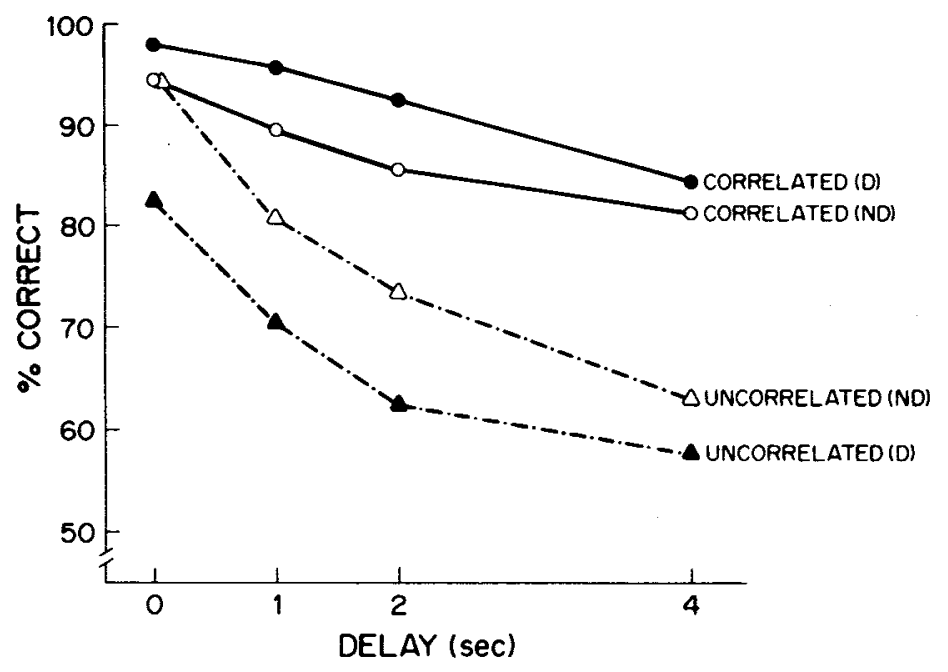

Figure 2. Percentage of correct choice responses by delay for each group during the mixed-delay retention tests with differential (D) outcomes in Experiment 1 and nondifferential (ND) outcomes in Experiment 2. 
Table 3

Sample-Response Latencies (in milliseconds) for High- and Low-Reinforcement-Probability Samples Averaged over the Last 10 Acquisition Sessions and the Last 10 MixedDelay Sessions of Experiment 1, and over the Last 10 Nondifferential Test Sessions of Experiment 2

\begin{tabular}{ccccc}
\hline Group & Probability & Acquisition & Mixed-Delay & Nondifferential \\
\hline Correlated & High & 230 & 274 & 248 \\
& Low & 483 & 615 & 293 \\
Uncorrelated & High & 284 & 311 & 351 \\
& Low & 779 & 1243 & 403 \\
\hline
\end{tabular}

the samples associated with the low reinforcement probability $(p=.2)$ than those associated with the high reinforcement probability $(p=1.0)$. ANOVAs on the latency data confirmed the overall difference for pecking highversus low-reinforcement-probability samples $\left[F_{\mathrm{S}}(1,6)=\right.$ 27.44 and 24.53 for acquisition and mixed-delay tests, respectively]. Although this difference appeared to be larger in Group Uncorrelated than in Group Correlated, the group $\times$ probability interactions fell short of significance $\left[F_{\mathrm{S}}(1,6)=2.85\right.$ and 5.30 for acquisition and testing, respectively].

\section{Discussion}

Experiment 1 replicates Honig et al.'s (1984) finding that differential outcomes facilitate conditional discrimination learning when they are correlated with discriminative responding to the comparisons relative to when they are uncorrelated with such responding. Honig et al. obtained this effect in successive (go/no-go) matching with food versus water outcomes. Here, the same result was obtained in two-alternative matching with different probabilities of the same (food) reinforcer. Experiment 1 also demonstrated that working memory performances following acquisition to criterion were considerably more accurate with correlated than with uncorrelated outcomes.

These results cannot be explained simply in terms of enhanced sample discriminability. If the only effect of the sample-outcome associations was to make the samples more distinctive (hence potentiating their individual control over choice), then performances by the two groups should have been comparable. The fact that they were not indicates that some other facior was involved.

One possibility, not heretofore mentioned, is that comparison discriminability was enhanced in Group Correlated, since the $R$ and $G$ alternatives for this group (but not Group Uncorrelated) were also associated with different reinforcement probabilities. But this account of the results seems implausible for two reasons. First, although MTS acquisition can be affected by comparison discriminability (Carter \& Eckerman, 1975; Urcuioli \& Zentall, 1986), there is no known corresponding effect on retention (Urcuioli \& Zentall, 1986; Urcuioli et al., 1989; Zentall, Urcuioli, Jagielo, \& Jackson-Smith, 1989). Second, and more importantly, comparison distinctiveness by itself cannot indicate which choice alternative the birds should select on any given trial. That information must come either from the samples or some other conditional cue.

A more plausible explanation attributes the betweengroup differences in performance to the validity of the outcome-expectancy cue. In Group Correlated, that cue was predictive of correct choice. In other words, choosing the red comparison in the presence of the certain expectation of food was always correct, as was choosing green in the presence of the uncertain expectation of food. By contrast, those relationships did not hold in Group Uncorrelated, since each comparison alternative was correct equally often in the presence of each expectancy. Thus, performances of the birds in that group could not possibly have been governed by the expectancies themselves, but had to be based instead on some other cue. The most likely candidate, obviously, was that provided by the sample stimuli.

Considering, then, that the correlated birds could match correctly on the basis of both the samples and their outcome expectancies, but that the uncorrelated birds could rely only on the samples, would there be a difference in the degree to which the common cue-the samplescontrolled their performances? The next experiment was designed to address this question.

\section{EXPERIMENT 2}

Measuring sample-stimulus control in the absence of any potentially confounding effect of differential-outcome expectancies requires that the latter be removed. This can presumably be accomplished by altering the sample-outcome associations such that both outcomes now follow each sample stimulus equally often. This was accomplished in Experiment 2 in the following manner. First, the MTS task was discontinued while birds in both groups received food reinforcement equally often following all center-key (sample) stimuli. In other words, differentialoutcome expectancies were initially "extinguished" off the matching baseline in the sense that they were replaced by nondifferential expectancies (Urcuioli, 1990). Then, many-to-one MTS was resumed but with correct choices now reinforced at the same probability on all trials. It was expected that performance during this nondifferential test phase would reflect how well the samples themselves are able to support accurate matching.

If differential-outcome expectancies provided a redundant cue for Group Correlated in Experiment 1, and that cue overshadowed the samples, then their samples should support less accurate matching during these tests than in Group Uncorrelated (Urcuioli, 1985; Wagner et al., 1968). On the other hand, if differential outcomes enhanced sample-stimulus control independently of their prior relationship to correct choice, then each group's test performance should be the same. Clearly, the latter result seems rather unlikely in view of the findings of Experiment 1 . But comparable levels of accuracy might also be anticipated if, say, outcome expectancies provided an ad- 
ditional but noncompeting source of conditional stimulus control in Group Correlated during Experiment 1 (Urcuioli, 1990).

\section{Method}

Subjects and Apparatus. The subjects and apparatus were the same as those used in Experiment 1.

Procedure. Following the last session of Experiment 1, each bird was returned for 10 sessions to the "pretraining" phase like that which had preceded acquisition. All four "sample" stimuli (V, H, $B$, and $Y$ ) appeared an equal number of times on the center key during these 60 -trial sessions. However, each stimulus was now followed by food with the same probability (.6), which was the average of the two probabilities (1.0 and .2) used in Experiment 1. All other procedural details, however, were identical to those previously described. The off-baseline phase ended after 10 sessions, because this was found to be sufficient to eliminate the differential responding previously observed to the hues and to the lines.

Next, the birds were run for 20 sessions on mixed-delay manyto-one MTS with correct choices reinforced equally often ( $p=.6$ ) on all trials (see Table 2). Other than the change to nondifferential outcomes, the matching procedure was exactly the same as that used during the mixed-delay tests of Experiment 1.

\section{Results}

By the end of nondifferential pretraining, the birds in both groups were responding as often and as quickly to all four "sample" stimuli. For example, the average latency to peck the formerly high- and low-probability samples differed by only 22 and $17 \mathrm{msec}$ in Groups Correlated and Uncorrelated, respectively, over the last five offbaseline sessions. Neither of these within-group latency differences was significant $[F \mathrm{~s}(1,3)=1.69$ and 0.18$]$.

The unfilled symbols in Figure 2 (see page 413 ) plot the retention gradient for each group over the last 10 nondifferential test sessions. The shift from differential (D) to nondifferential (ND) outcomes produced a drop in accuracy in Group Correlated, but an increase in Group Uncorrelated. Nonetheless, Group Correlated continued to match more accurately over longer delays than did Group Uncorrelated. This between-group difference was confirmed by analysis of variance, which revealed significant effects of group $[F(1,6)=7.62]$ and delay $[F(3,18)=$ 27.89 , as well as a significant group $\times$ delay interaction $[F(3,18)=4.56]$.

Figure 3 shows how performance changed in each group immediately after the shift to nondifferential outcomes. Here, retention gradients for the first 10 nondifferential (ND) test sessions are plotted against those for the last 10 differential (D) sessions in Experiment 1. The immediate effect of shifting to nondifferential outcomes in Group Correlated was to produce a rather substantial drop in accuracy. By contrast, the shift had little, if any, immediate effect on performance in Group Uncorrelated. An ANOVA on each group's data supported these conclusions. For Group Correlated, the effect of outcome condition (differential vs. nondifferential) was significant $[F(1,3)=10.90]$, as was the overall delay effect $[F(3,9)=7.53]$. The outcome $\times$ delay interaction was not significant, however $[F(3,9)=1.56]$. For Group Uncorrelated, only the overall effect of delay was significant $[F(3,9)=87.04]$

Finally, the right-hand column of Table 3 shows that, for both groups, sample-response latencies for the last 10 nondifferential test sessions were quite similar. Although the average latency to peck the samples previously associated with the high reinforcement probability was slightly less than the corresponding latency to peck the samples previously associated with the low reinforcement probability, the difference was not significant in either group $[F \mathrm{~s}(1,3)=1.01$ and 1.74 for Groups Correlated and Uncorrelated].

\section{Discussion}

Two notable results were obtained in this experiment. First, shifting to nondifferential outcomes produced a siz-

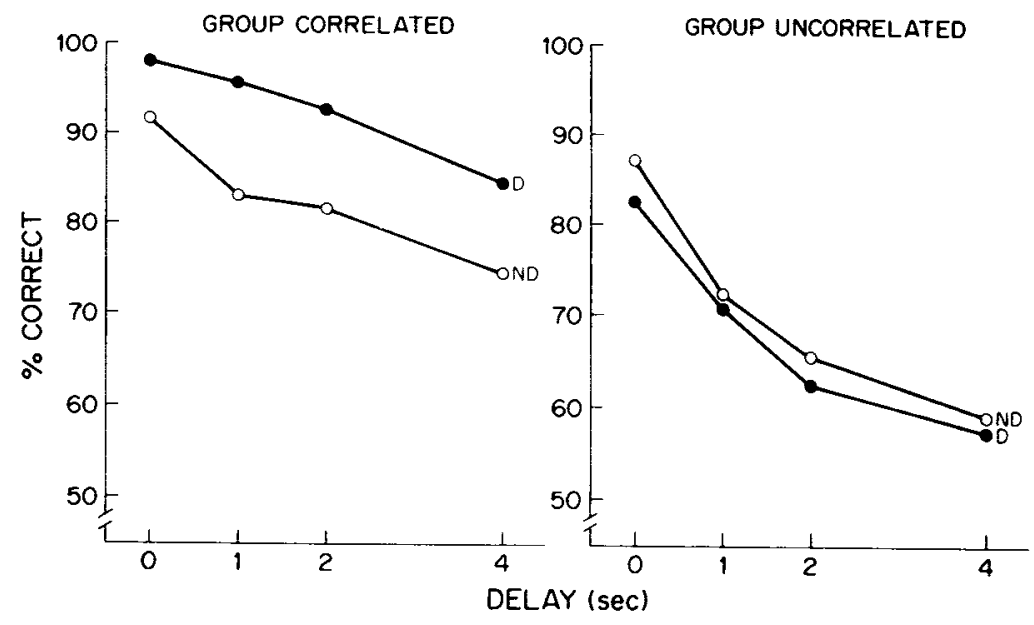

Figure 3. Retention gradients for each group from the last 10 differential-outcome (D) sessions in Experiment 1 and the first 10 nondifferential-outcome (ND) sessions in Experiment 2. 
able drop in matching accuracy in Group Correlated, especially during initial testing. Second, despite the drop in performance, Group Correlated continued to match more accurately on mixed-delay MTS than did Group Uncorrelated.

The drop in Group Correlated's performance cannot be explained simply in terms of generalization decrement produced by the change in outcomes for correct choice. After all, those outcomes also changed for Group Uncorrelated, yet their performances were not similarly affected. Instead, the results are more consistent with the idea that (1) prior differential-outcomes training had provided the correlated birds with a valid outcome-expectancy cue, and (2) the shift to nondifferential outcomes removed that cue. Consequently, these birds now had to choose between the comparisons on the basis of the samples alone. Although the samples continued to support accurate matching, the level of performance they supported by themselves was lower than that observed when outcome expectancies could provide a redundant cue for choice.

Interestingly, those expectancies did not overshadow the samples. This was shown by the finding that matching accuracy during nondifferential testing was not any lower in Group Correlated than in Group Uncorrelated. On the contrary, it was higher. This indicates that, far from being diminished, sample-stimulus control had apparently been enhanced by the differential outcomes in Group Correlated. This result is not anticipated, however, by the sample discriminability hypothesis described earlier. After all, the samples in both groups were originally associated with different outcomes, so why should conditional stimulus control by the samples be any stronger in one group than in the other?

One possible explanation is that the results do not actually reflect differences in sample-stimulus control but, rather, the persistence of differential-outcome expectancies despite the shift to nondifferential outcomes. But this seems unlikely for a variety of reasons. First, the immediate and sizeable drop in Group Correlated's accuracy during testing is a clear indication that there was indeed a change in the factors controlling matching performance (Peterson, Wheeler, \& Armstrong, 1978). Second, sampleresponse latencies in each group were quite similar, unlike what they had been when outcomes were differential with respect to the samples. To the extent that these latencies accurately (and independently) reflect outcome expectancies, they too suggest that those expectancies were no longer differential with respect to the samples. Finally, 20 test sessions with nondifferential reinforcement preceded by 10 "pretraining" sessions in which all samples were followed by food equally often seem to be more than sufficient to eliminate any previously learned, differential expectancies.

Another explanation of the differences in test performance is that, given their faster acquisition and better working memory performances in Experiment 1, the correlated birds had been reinforced more often than the uncorrelated birds for choosing correctly after each sample stimulus. In other words, stronger sample-stimulus control in Group Correlated was simply a by-product of how frequently their choices had been reinforced during original, differential-outcomes training. If so, then considering that this group continued to experience more frequent reinforcement than Group Uncorrelated during the nondifferential test, its "advantage"' in sample-stimulus control should carry forward into future conditions. However, if the difference in sample-stimulus control is a product of the prior relationship between outcomes and correct choice, then shifting birds from one differential-outcomes condition to the other should eliminate, or perhaps even reverse, this difference. These contrasting predictions were tested in Experiment 3.

\section{EXPERIMENT 3}

In this experiment, all pigeons were initially retrained on many-to-one MTS with the different reinforcement probabilities used in Experiment 1. However, pigeons originally trained in the correlated condition were now shifted to the uncorrelated condition, and vice versa. After reacquisition with 0 -sec delays and subsequent mixeddelay training, the MTS task was once again temporarily discontinued while the birds received off-baseline nondifferential reinforcement for responding to each sample stimulus. The birds were then tested on mixed-delay MTS with nondifferential outcomes for one final assessment of their ability to match on the basis of the samples alone.

\section{Method}

Subjects and Apparatus. The subjects and apparatus were the same as those used in the preceding two experiments.

Procedure. This experiment consisted of four phases described below in the order in which they occurred.

Differential-outcomes pretraining. Following the last MTS session in Experiment 2, all birds received 10 sessions of off-baseline " "pretraining" with different probabilities of food reinforcement for pecking the center-key (sample) stimuli. These sessions were designed to help reestablish differential-outcome expectancies to the samples and to do so in such a way that the predictive relationship between those expectancies and choice in the subsequent MTS phase (see below) would be the opposite of what it had been in Experiment 1. For two of the original pairs of correlated and uncorrelated birds, the first five of these sessions involved only the $V$ and $\mathrm{H}$ lines. For the other two pairs, only the $\mathrm{B}$ and $\mathrm{Y}$ hues appeared. One stimulus always ended in food reinforcement $(p=1.0)$, while the other ended in food only $20 \%$ of the time $(p=.2)$. Furthermore, the relationship of stimulus to reinforcement probability was the reverse of what it had been in Experiment 1 .

The last five pretraining sessions involved all four "sample" stimuli (V, H, B, and Y). The two stimuli that appeared during the first five sessions continued to be associated with the different (but reversed) probabilities of food reinforcement. The other two stimuli were also associated with different reinforcement probabilities (1.0 vs. .2), but these were the same as they had been in Experiment 1 . In all other respects, the 10 off-baseline sessions were identical to those described in the preceding experiments.

Many-to-one MTS with differential outcomes. Next, the birds were retrained on many-to-one MTS in which the probability of reinforcement for correct choice differed across sample trial types 


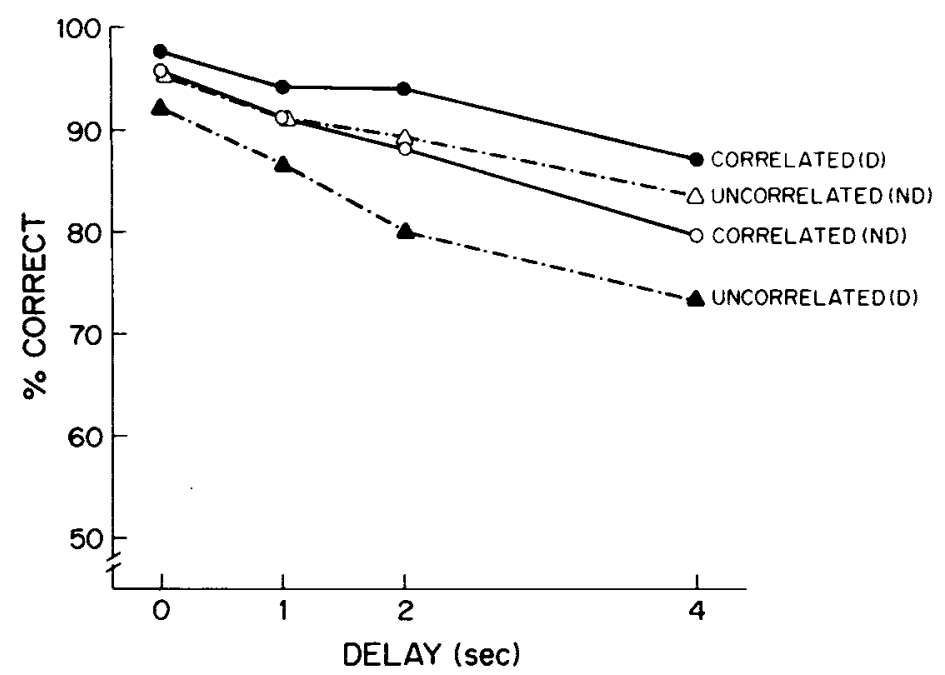

Figure 4. Percentage of correct choice responses by delay for each group during the mixed-delay retention tests with differential (D) and nondifferential (ND) outcomes in Experiment 3. Note that the group designations reflect the training conditions following outcome reversal and are the opposite of what they were in Experiment 1.

(see Table 2). For two of the samples, these probabilities were reversed relative to Experiment 1. The net result of this change was that outcomes previously correlated with correct choice were now uncorrelated with those choices, and vice versa. The birds in each group were trained under these new conditions with 0 -sec delays until they matched correctly on $90 \%$ or more of all trials for five of six consecutive sessions. They then received 20 additional sessions with mixed delays (i.e., $0,1,2$, and $4 \mathrm{sec}$ ).

Nondifferential-outcomes pretraining. Following the last mixeddelay session, the MTS procedure was again discontinued while the birds received nondifferential reinforcement for pecking the four center-key (sample) stimuli. During these 10 off-baseline sessions, pecking each stimulus produced food $60 \%$ of the time $(p=.6)$. Otherwise, the procedural details were the same as those previously described for nondifferential pretraining (see Experiment 2).

Many-to-one MTS with nondifferential outcomes. In the final phase, the birds were retested for 20 sessions on mixed-delay manyto-one MTS with the same probability of reinforcement $(p=.6)$ following all correct choices. These sessions provided a measure of sample-stimulus control following the change from correlated to uncorrelated outcomes, and vice versa.

\section{Results}

Despite the switch in conditions, reacquisition of 0 delay many-to-one MTS with differential outcomes proceeded very rapidly in both groups. The average number of sessions necessary to complete the reacquisition phase was $\mathbf{5 . 5}$ for the group shifted from the correlated to uncorrelated condition versus 7.5 for the group shifted in the opposite direction. This difference was not statistically significant $[F(1,6)=3.00]$.

The filled symbols in Figure 4 plot the retention performances of each group over the last 10 mixed-delay sessions that followed MTS reacquisition with differential (D) outcomes. These results mirror those obtained in Experi-

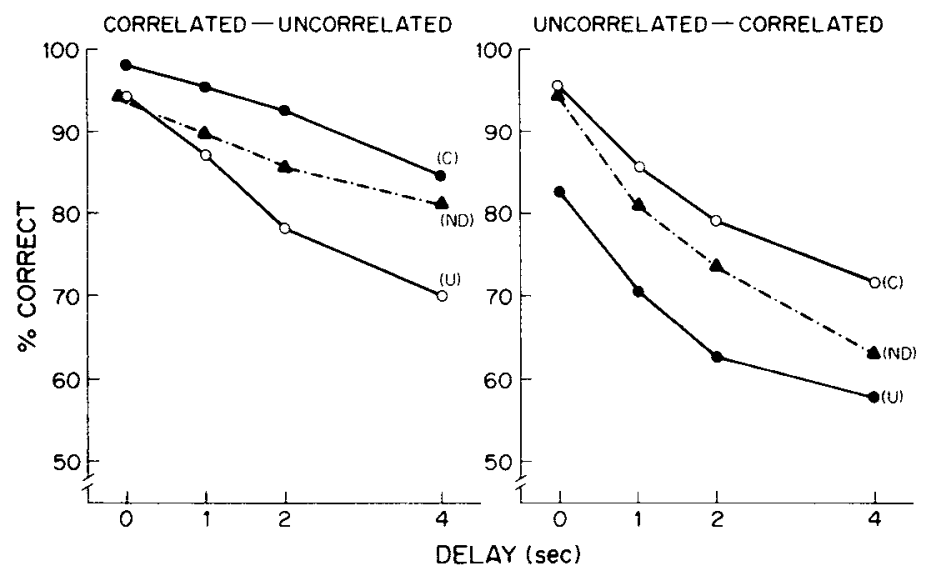

Figure 5. Retention gradients for each group from the last $\mathbf{1 0}$ sessions of Experiments 1 and 2 (filled symbols) and the first 10 differential-outcome sessions of Experiment 3 (open symbols). See text for further details. 
ment 1 . Delayed matching accuracy was higher overall, and the rate of forgetting was slower, for birds trained with correlated rather than uncorrelated outcomes. Analysis of variance revealed a significant group effect $[F(1,6)=63.92]$, a significant group $\times$ delay interaction $[F(3,18)=2.22]$, and a significant overall effect of delay $[F(3,18)=19.34]$.

Figure 5 (on page 417) shows how performance in each group was affected by the shift in conditions. In this figure, matching accuracy over the first 10 mixed-delay (differential-outcome) sessions in this experiment are plotted against performances over (1) the last 10 nondifferential sessions of Experiment 2, and (2) the last 10 differential-outcome sessions of Experiment 1. Data from the birds shifted from the correlated to the uncorrelated condition are shown in the left panel. The corresponding data from the birds shifted in the opposite direction are shown in the right panel.

Despite the different orders in which the two differential-outcome conditions were received, the overall pattern of results is identical for the two groups. Delayed matching accuracy was highest when outcomes were correlated $(C)$ with correct choice, lowest when they were uncorrelated $(\mathrm{U})$, and intermediate when food occurred equally often following all choices (ND, nondifferential). Compared to the immediately preceding (i.e., ND) condition, the group shifted to uncorrelated outcomes showed a drop in matching accuracy, while the group shifted to correlated outcomes performed more accurately.

An ANOVA confirmed the latter two effects. For the birds shifted from correlated to uncorrelated training, matching was less accurate overall, and it showed a greater drop with uncorrelated as opposed to nondifferential outcomes as delays increased $[F(1,3)=13.39$, and $F(3,9)=4.88$, respectively]. This occurred despite the fact that uncorrelated training followed the nondifferential phase. For the birds shifted from uncorrelated to correlated training, overall performance in the latter condition did not differ significantly from that observed at the end of the preceding nondifferential phase $[F(1,3)=$ 4.05]. However, the interaction between condition and delay was significant $[F(3,9)=3.32]$. Subsequent randomized-blocks ANOVAs at each delay (using an error mean square averaged across delays) revealed that this group matched more accurately at the 4-sec delay with correlated (differential) outcomes than it did with nondifferential outcomes $[F(1,12)=8.91]$.

The open symbols in Figure 4 show the results from the nondifferential (ND) test that followed the shift in conditions. In contrast to Experiment 2, where there was a large advantage in favor of the correlated birds, the two groups now performed similarly when all correct choices were reinforced equally often. Nonetheless, just as it did in Experiment 2 (cf. Figure 2), the change to nondifferential outcomes following correlated training produced a sizeable drop in matching accuracy, but enhanced matching accuracy following uncorrelated training.

A between-groups ANOVA on the nondifferential data from this experiment revealed that only the delay effect was significant $[F(3,18)=14.87]$. Separate within-group ANOVAs were then run to compare how accurately each group matched on the nondifferential test relative to its immediately preceding differential-outcomes phase. For the correlated group, overall accuracy in these two conditions did not significantly differ $[F(1,3)=5.89]$. However, there was a significant interaction of condition with delay $[F(3,9)=5.64]$. Subsequent randomizedblocks ANOVAs at each delay using an average error mean square showed that this group matched less accurately at the 4-sec delay with nondifferential outcomes than it did with differential outcomes $[F(1,12)=11.25]$. For the uncorrelated group, matching performance was significantly more accurate with nondifferential than with differential outcomes $[F(1,3)=14.89]$. The interaction between outcome condition and delay was significant as well $[F(3,9)=2.90]$.

Figure 6 shows the immediate effect of the change to nondifferential outcomes following the switch between

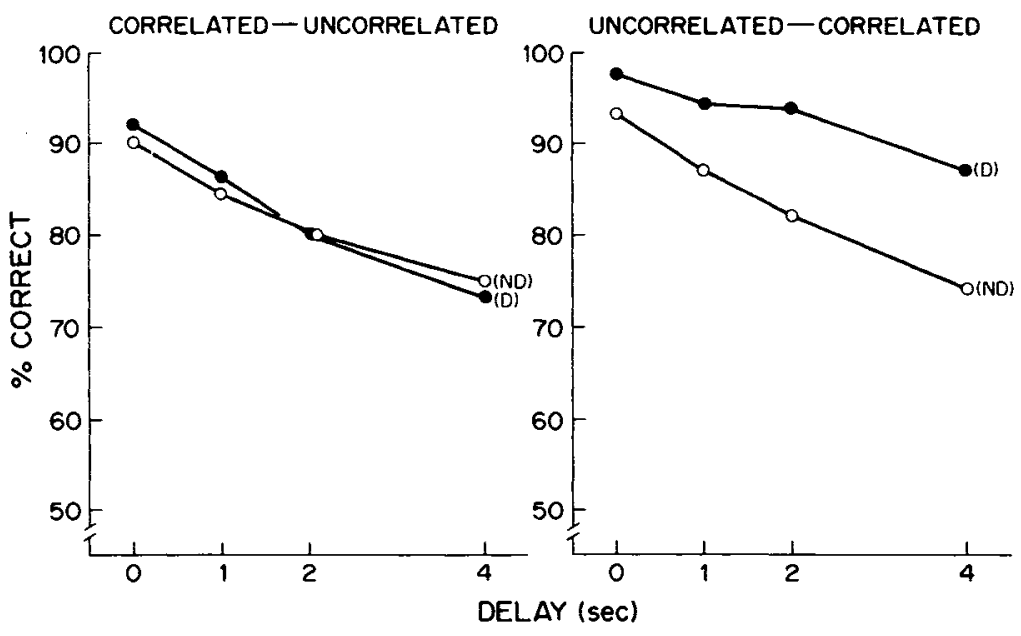

Figure 6. Retention gradients for each group from the last 10 differential-outcome (D) sessions and the first 10 nondifferential-outcome (ND) sessions in Experiment 3. 
Table 4

Sample-Response Latencies (in milliseconds) for High- and LowReinforcement-Probability Samples Averaged over the Last 10 Sessions of Differential-Outcomes Training and the Last 10 Nondifferential Test Sessions of Experiment 3

\begin{tabular}{cccc}
\hline & & \multicolumn{2}{c}{ Outcomes } \\
\cline { 3 - 4 } Group & Probability & Differential & Nondifferential \\
\hline Correlated-Uncorrelated & High & 207 & 228 \\
& Low & 558 & 253 \\
Uncorrelated-Correlated & High & 283 & 324 \\
& Low & 1233 & 444 \\
\hline
\end{tabular}

correlated and uncorrelated training. Each group's performance over the first 10 nondifferential (ND) sessions are plotted against its performance over the last 10 differential-outcome (D) sessions. Once again, the results are highly similar to those obtained in Experiment 2. Accuracy of performance dropped substantially when nondifferential testing followed correlated training (right panel; cf. left panel of Figure 3). By contrast, matching accuracy was relatively unaffected when prior many-toone MTS involved uncorrelated outcomes (left panel; cf. right panel of Figure 3).

ANOVAs on these data showed that matching performance was significantly less accurate, and decreased more rapidly with increasing delays, with nondifferential as opposed to differential outcomes in the uncorrelated-correlated group $[F(1,3)=23.64$ and $F(3,9)=$ 6.50 , respectively]. By contrast, neither the effect of condition nor the interaction of condition with delay was significant in the correlated-uncorrelated group $\left(F_{\mathrm{s}}<.57\right)$.

Table 4 shows each group's average latency to peck the high- versus low-reinforcement-probability samples during the differential- and nondifferential-outcome phases of this experiment. (In the latter condition, "high" and "low" refer to the samples' prior association with reinforcement). With differential outcomes, both groups responded more quickly to the samples signaling the 1.0 probability than to those signaling the 0.2 probability
$[F(1,6)=14.17]$. Neither the group effect nor the group $\times$ probability interaction was significant $\left[F_{\mathrm{s}}(1,6)=2.73\right.$ and 2.94 , respectively]. These latency differences were reduced considerably when the probability of reinforcement was the same on all trials $(p=0.6)$. An ANOVA on the data from the latter, nondifferential condition revealed no significant difference in latency to peck, no effect of group, and no group $\times$ probability interaction $[F \mathrm{~s}(1,6)=3.88,3.65$, and 1.66 , respectively $]$.

\section{Discussion}

The primary purpose of this experiment was to see whether or not the between-group differences found in Experiments 1 and 2 would be reversed or eliminated if the birds in each group were shifted to the opposite differential-outcomes condition. The shift did reverse the performance differences when correct choices were reinforced by different probabilities of food, and it produced within-group effects that mimicked the between-group ones. For example, delayed matching by each group was less accurate with uncorrelated than with correlated outcomes, independent of the order in which these conditions were received. Also, despite extensive prior training on many-to-one MTS, the shift to uncorrelated outcomes in Group Correlated-Uncorrelated caused matching accuracy to initially drop below the level observed in each of its prior two conditions. The latter finding supports the idea that comparison choice in this group was initially governed in part by differential outcome expectancies (viz., in Experiment 1). When those expectancies were present once again but were no longer predictive of correct choice, they interfered with matching performances. By contrast, differential-outcome expectancies facilitated performance when they became predictive of choice, as the data from Group Uncorrelated-Correlated show.

The finding that the performances of the two groups during the nondifferential test of this experiment were now comparable indicates that the shift also affected conditional

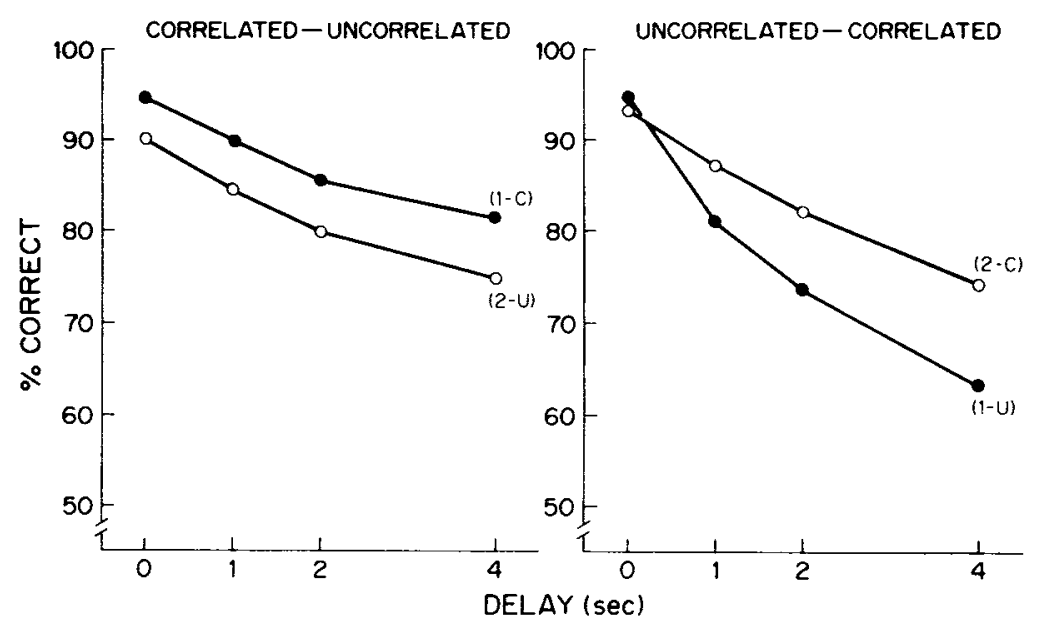

Figure 7. Retention gradients for each group from the first (1) and second (2) nondifferential test conducted after correlated $(C)$ or uncorrelated $(U)$ training. See text for further details. 
stimulus control by the samples. Thus, the prior betweengroup difference in nondifferential performance (cf. Figure 2) does not appear to be due simply to the cumulative frequency with which each sample-comparison combination had been reinforced.

Further support for this conclusion is provided in Figure 7 , which compares matching accuracy at the beginning of the second nondifferential test with that seen at the end of the first nondifferential test. For Group Correlated-Uncorrelated, the mean level of performance at the beginning of the second test (i.e., the one that followed uncorrelated training, 2-U) was somewhat lower than the mean level of performance at the end of the first test (i.e., the one that followed correlated training, 1-C), although the effect was not statistically significant $[F(1,3)=1.52]$. Nevertheless, it was clearly evident in 3 of the 4 birds. By contrast, 3 of the 4 Group Uncorrelated-Correlated birds showed a change in the opposite direction. They matched more accurately on the nondifferential test that followed correlated training (2-C) than on the one that followed uncorrelated training (1-U). In this group, there was a significant test $X$ delay interaction $[F(3,9)=3.79]$. Together, these data suggest that samplestimulus control was influenced by the immediately prior relationship between outcomes and correct choice.

\section{GENERAL DISCUSSION}

The current study, like that of Honig et al. (1984), was designed to assess the relative contributions of outcome expectancies and enhanced sample discriminability to the DOE. The results from this study, in which two-choice conditional discriminations were used, are consistent with the Honig et al. findings in successive matching, and add considerably to them. First, differential outcomes were shown to produce faster MTS acquisition when the outcomes were correlated with correct comparison choice than when they were uncorrelated with those choices. Second, correlated outcomes also produced substantially better retention than uncorrelated outcomes after initial acquisition to high levels of accuracy. Third, the retention effect was reproduced when birds in each group were later shifted to the opposite differential-outcomes condition. Fourth, the within-group effect of correlated versus uncorrelated training paralleled the between-group retention differences. Finally, conditional stimulus control by the samples was found to be stronger following correlated than following uncorrelated training, at least after initial exposure to these conditions.

Most of these results are more easily accommodated by outcome-expectancy mediation than by enhanced sample discriminability. After all, if the sole consequence of the associations between samples and different end-of-trial reinforcement probabilities was simply to enhance the distinctiveness of the samples, then acquisition and retention of many-to-one MTS should have been similar in the correlated and uncorrelated conditions. But that was not the case in either the between- or within-group compari- sons. Clearly, the different outcomes had to be doing something more than simply potentiating the control exerted by the individual samples over pigeons' choices. Considering the already abundant evidence that sample-outcome associations can generate another discriminative cue for performance (i.e., an outcome-expectancy cue-see Edwards et al., 1982; Peterson, 1984; Urcuioli, 1990), the data suggest instead that the differences between conditions reflect the added control exerted by that cue in the correlated condition. This analysis is also supported by the results obtained from the shift manipulation and (at least in part) from the nondifferential tests.

For example, when a previously valid outcome-expectancy cue was made irrelevant by changing from correlated to uncorrelated outcomes, matching accuracy dropped. Conversely, when a previously irrelevant cue became predictive of correct choice by shifting birds in the opposite direction, choice accuracy increased (see Figure 5). Similarly, changing from correlated to nondifferential outcomes produced an immediate and sizeable drop in accuracy, as anticipated if such a change removed one of the cues formerly controlling performance (i.e., differential-outcome expectancies). By contrast, the corresponding change after uncorrelated training did not have the same effect-nor should it have, if differentialoutcome expectancies were not providing a cue for choice in the first place (see Figures 3 and 6).

Nonetheless, the outcome-expectancy explanation cannot easily account for the advantage that the correlated birds continued to show during the first nondifferential test. With the presumed outcome-expectancy cue gone and only the samples to guide performance, this analysis would predict that the correlated birds would show, at best, the same level of accuracy as that of the uncorrelated birds (Urcuioli, 1990). At worst, their performances should be less accurate than the uncorrelated subjects', assuming that the expectancy cue had overshadowed their samples (Mackintosh, 1983; Rescorla \& Wagner, 1972; Wagner et al., 1968). Consequently, the finding that they were more accurate in their test performances was quite surprising, especially since in a previous study (Urcuioli, 1985) evidence had been found for overshadowing in an analogous many-to-one task.

In that earlier study, pigeons taught to produce the comparisons by responding differentially to the sample stimuli showed weaker control by the samples themselves when their differential sample behaviors had been correlated with the correct choices than when they had been uncorrelated with those choices. In the present study, birds also responded differentially to the samples during differentialoutcomes training (i.e., they pecked the high-reinforcement-probability samples sooner than the low-reinforcement-probability samples), yet there was no sign of a similar overshadowing effect in the correlated group. One explanation for this discrepancy might be that the different response latencies did not provide a salient enough cue to overshadow the samples (Miles \& Jenkins, 1973; see also Urcuioli, 1990). But that explanation is inade- 
quate, because it predicts the same degree of samplestimulus control in the two groups, not the finding that such control was stronger in the correlated group.

The latter result might be related to the finding, recently reported by Urcuioli et al. (1989, Experiment 3), that pigeons commonly code samples that are associated with the same correct comparison in many-to-one MTS. In that study, pigeons initially trained on many-to-one MTS were later taught to match two of the original samples to new comparison alternatives. Then, during testing, the remaining two samples were substituted for those used during the interim phase. Urcuioli et al. observed positive transfer of performance when samples associated with the same comparisons in the prior many-to-one task replaced one another. Conversely, negative transfer occurred when the substitution involved associatively different samples. Apparently, the mapping of multiple samples onto common comparisons in many-to-one MTS made those samples functionally equivalent (viz., they were "commonly coded"').

Perhaps the presence versus absence of common coding can explain the nondifferential test results obtained in Experiment 2 (see Figure 2). For example, since the samples that were associated with the same correct comparison were also associated with the same reinforcing outcome in Group Correlated, this may have encouraged common coding. By contrast, the effect may have been discouraged in Group Uncorrelated because the samples with common comparison associates had different outcome associates.

This account is admittedly quite speculative, and it leaves a lot to be explained (e.g., why the nondifferential test results of Urcuioli, 1985, were the opposite of those obtained here). It is also unclear why the shift from correlated to uncorrelated outcomes (and vice versa) did not reverse the group differences seen during the initial nondifferential test (see Figures 2 and 4). Still, many aspects of the data are consistent with this interpretation. If correct, it means that differential outcomes can have other effects on conditional discrimination performance besides generating outcome expectancies. It is certainly the case, however, that the relationship of those outcomes with correct choice in many-to-one MTS plays a powerful role in determining the sorts of events that will govern pigeons' matching performance.

\section{REFERENCES}

Brodigan, D. L., \& Peterson, G. B. (1976). Two-choice conditional discrimination performance of pigeons as a function of reward expectancy, prechoice delay, and domesticity. Animal Learning \& Behavior, 4, 121-124.

Carter, D. E., \& Eckerman, D. A. (1975). Symbolic matching by pigeons: Rates of learning complex discriminations predicted from simple discriminations. Science, 187, 662-664.

DELONG, R. E., \& WASSERMAN, E. A. (1981). Effects of differential reinforcement expectancies on successive matching-to-sample perfor- mance in the pigeon. Journal of Experimental Psychology: Animal Behavior Processes, 7, 394-412

Edwards, C. A., Jagielo, J. A., Zentall, T. R., \& Hogan, D. E. (1982). Acquired equivalence and distinctiveness in delayed matching to sample by pigeons: Mediation by reinforcer-specific expectancies. Joumal of Experimental Psychology: Animal Behavior Processes, 8, 244-259.

Honig, W. K., Matheson, W. R., \& Dodd, P. W. D. (1984). Outcome expectancies as mediators for discriminative responding. Canadian Journal of Psychology, 38, 196-217.

HoniG, W. K., \& Thompson, R. K. R. (1982). Retrospective and prospective processing in animal working memory. In $\mathrm{G}$. H. Bower (Ed.), The psychology of learning and motivation (Vol. 16, pp. 239282). New York: Academic Press.

LAWRENCE, D. H. (1949). Acquired distinctiveness of cues: I. Transfer between discriminations on the basis of familiarity with the stimulus. Journal of Experimental Psychology, 39, 770-784

Linwick, D., Overmier, J. B., Peterson, G. B., \& Mertens, M. (1988). Interaction of memories and expectancies as mediators of choice behavior. American Journal of Psychology, 101, 313-334.

MaCKINTOSH, N. J. (1983). Conditioning and associative learning. New York: Oxford University Press.

Miles, C. G., \& JeNkins, H. M. (1973). Overshadowing in operant conditioning as a function of discriminability. Learning \& Motivation, 4, 11-27.

Nelson, K. R., \& Wasserman, E. A. (1978). Temporal factors influencing the pigeon's successive matching-to-sample performance: Sample duration, intertrial interval, and retention interval. Joumal of the Experimental Analysis of Behavior, 30, 153-162.

Peterson, G. B. (1984). How expectancies guide behavior. In H. L. Roitblat, T. G. Bever, \& H. S. Terrace (Eds.), Animal cognition (pp. 135-148). Hillsdale, NJ: Erlbaum.

Peterson, G. B., Linwick, D., \& Overmier, J. B. (1987). On the comparative efficacy of memories and expectancies as cues for choice behavior in pigeons. Learning \& Motivation, 18, 1-20.

Peterson, G. B., \& Trapold, M. A. (1982). Expectancy mediation of concurrent conditional discriminations. American Journal of Psychology, 95, 571-580.

Peterson, G. B., Wheeler, R. L., \& Armstrong, G. D. (1978). Expectancies as mediators in the differential-reward conditional discrimination performance of pigeons. Animal Learning \& Behavior, 6 , 279-285.

Peterson, G. B., Wheeler, R. L., \& Trapold, M. A. (1980). Enhancement of pigeon's conditional discrimination performance by expectancies of reinforcement and nonreinforcement. Animal Learning \& Behavior, 8, 22-30.

Rescorla, R. A., \& WAGner, A. R. (1972). A theory of Pavlovian conditioning: Variations in the effectiveness of reinforcement and nonreinforcement. In A. H. Black \& W. F. Prokasy (Eds.), Classical conditioning II: Current research and theory (pp. 64-99). New York Appleton-Century-Crofts.

RODGER, R. S. (1975). The number of non-zero, post hoc contrasts from ANOVA and error rate: I. British Joumal of Mathematical \& Statistical Psychology, 28, 71-78.

SANTI, A., \& RoberTs, W. A. (1985a). Prospective representation: The effects of varied mapping of sample stimuli to comparison stimuli and differential trial outcomes on pigeons' working memory. Animal Leaming \& Behavior, 13, 103-108.

SANTI, A., \& RoBERTS, W. A. (1985b). Reinforcement expectancy and trial spacing effects in delayed matching-to-sample by pigeons. Animal Learning \& Behavior, 13, 274-284.

TRAPOLD, M. A. (1970). Are expectancies based upon different positive reinforcing events discriminably different? Learning \& Motivation, 1, 129-140.

URCUIOLI, P. J. (1985). On the role of differential sample behaviors in matching-to-sample. Journal of Experimental Psychology: Animal Behavior Processes, 11, 502-519.

UrCuroli, P. J. (1990). Some relationships between outcome expec- 
tancies and sample stimuli in pigeons' delayed matching. Animal Leaming \& Behavior, 18, 302-314.

URCUIOLI, P. J., \& ZENTALL, T. R. (1986). Retrospective coding in pigeons' delayed matching-to-sample. Joumal of Experimental Psychology: Animal Behavior Processes, 12, 69-77.

Urcuioli, P. J., \& ZeNTALL, T. R. (1990). On the role of trial outcomes in delayed discriminations. Animal Learning \& Behavior, 18, 141-150.

Urcuioli, P. J., Zentall, T. R., Jackson-Smith, P., \& Steirn, J. N. (1989). Evidence for common coding in many-to-one matching: Retention, intertrial interference, and transfer. Joumal of Experimental Psychology: Animal Behavior Processes, 15, 264-273.

Wagner, A. R., Logan, F. A., Haberlandt, K., \& Price, T. (1968). Stimulus selection in animal discrimination learning. Joumal of $E x-$ perimental Psychology, 76, 171-180.

Williams, D. A., Butler, M. M., \& Overmier, J. B. (1990). Expec- tancies of reinforcer location and quality as cues for a conditional discrimination in pigeons. Joumal of Experimental Psychology: Animal Behavior Processes, 16, 3-13.

Wright, A. A., \& SANDs, S. F. (1981). A model of detection and decision processes during matching-to-sample by pigeons: Performance with 88 different wavelengths in delayed and simultaneous matching tasks. Journal of Experimental Psychology: Animal Behavior Processes, 7, 191-216.

Zentall, T. R., Urcuiol, P. J., Jagielo, J. A., \&ackson-Smith, P. (1989). Interaction of sample dimension and sample-comparison mapping on pigeons' performance of delayed conditional discriminations. Animal Learning \& Behavior, 17, 172-178.

(Manuscript received June 6, 1989;

revision accepted for publication June 3, 1990.) 